\title{
Risk Factors Associated with COVID-19 Hospitalization and Mortality: A Large Claims-Based Analysis Among People with Type 2 Diabetes Mellitus in the United States
}

\author{
Kristina S. Boye - Elif Tokar Erdemir - Nathan Zimmerman - Abraham Reddy - Brian D. Benneyworth · \\ Matan C. Dabora · Emily R. Hankosky · M. Angelyn Bethel · Callahan Clark · Cody J. Lensing · Scott Sailer • \\ Ramira San Juan · Robert J. Heine · Lida Etemad
}

Received: April 28, 2021 / Accepted: June 28, 2021 / Published online: July 18, 2021

(C) The Author(s) 2021

\section{ABSTRACT}

Introduction: Diabetes has been identified as a high-risk comorbidity for COVID-19 hospitalization. We evaluated additional risk factors for COVID-19 hospitalization and in-hospital mortality in a nationwide US database.

Methods: This retrospective study utilized the UnitedHealth Group Clinical Discovery Database (January 1, 2019-July 15, 2020) containing de-identified nationwide administrative claims, SARS-CoV-2 laboratory test results, and COVID19 inpatient admissions data. Logistic regression was used to understand risk factors for hospitalization and in-hospital mortality among people with type 2 diabetes (T2D) and in the overall population. Robustness of associations was further confirmed by subgroup and sensitivity analyses in the T2D population.

Supplementary Information The online version contains supplementary material available at https:// doi.org/10.1007/s13300-021-01110-1.

K. S. Boye · B. D. Benneyworth · M. C. Dabora

E. R. Hankosky · M. A. Bethel · R. J. Heine

Eli Lilly and Company, Indianapolis, IN, USA

E. Tokar Erdemir $(\bowtie) \cdot$ N. Zimmerman - A. Reddy ·

C. Clark - C. J. Lensing · S. Sailer · R. San Juan ·

L. Etemad

OptumLabs at UnitedHealth Group, Minneapolis,

MN, USA

e-mail: eliftokar@gmail.com
Results: A total of 36,364 people were identified who were either SARS-CoV-2 ${ }^{+}$or hospitalized for COVID-19. T2D was associated with increased COVID-19-related hospitalization and mortality. Factors associated with increased hospitalization risk were largely consistent in the overall population and the T2D subgroup, including age, male sex, and these top five comorbidities: dementia, metastatic tumor, congestive heart failure, paraplegia, and metabolic disease. Biguanides (mainly metformin) were consistently associated with lower odds of hospitalization, whereas sulfonylureas and insulins were associated with greater odds of hospitalization among people with T2D.

Conclusion: In this nationwide US analysis, T2D was identified as an independent risk factor for COVID-19 complications. Many factors conferred similar risk of hospitalization across both populations; however, particular diabetes medications may be markers for differential risk. The insights on comorbidities and medications may inform population health initiatives, including prevention efforts for high-risk patient populations such as those with T2D.

Keywords: Claim-based analysis; COVID-19; Risk factors; SARS-CoV-2; Type 2 diabetes 


\section{Key Summary Points}

Why carry out this study?

The clinical and demographic characteristics as well as specific risk factors of patients with diabetes with severe forms of COVID-19 in the US have not been extensively studied.

This study aimed to characterize additional risk factors for COVID-19 hospitalization and in-hospital mortality among those with type 2 diabetes mellitus (T2DM) and the overall population in a nationwide US database.

\section{What was learned from the study?}

Factors associated with increased hospitalization risk were largely consistent in the overall population and the T2D subgroup, including age, male sex, and these top five comorbidities: dementia, metastatic tumor, congestive heart failure, paraplegia, and metabolic disease.

Findings of this study further support known at-risk populations and can help guide risk stratification efforts across population health strategies (e.g., vaccine prioritization or targeted outreach campaigns).

\section{INTRODUCTION}

Coronavirus disease-2019 (COVID-19) is caused by the severe acute respiratory syndrome coronavirus-2 (SARS-CoV-2) virus, which was first identified in December 2019 during an outbreak of respiratory illness cases in Wuhan City, Hubei Province, China [1]. The disease subsequently spread to other countries, and on March 11, 2020, the World Health Organization declared COVID-19 a global pandemic [2]. As of February 2, 2021, a total of 102 million confirmed cases and 2.21 million deaths had been reported worldwide, with 25.6 million cases and 433,173 deaths recorded in the US alone [3].

The clinical manifestations of COVID-19 are heterogeneous. A majority of individuals experience only mild symptoms of fever, dry cough, and myalgia at presentation [4]. However, patients with severe disease may develop dyspnea and hypoxemia within 1 week after disease onset, which may deteriorate rapidly to acute respiratory distress syndrome or multiple organ failure, leading to death $[4,5]$. Older age and comorbidities, such as diabetes, chronic lung disease, hypertension, obesity, and other cardiovascular and cerebrovascular diseases, are common factors associated with hospitalization and mortality in patients with COVID-19 [6-13].

Diabetes is well described as a major comorbidity of hospitalized patients with COVID-19 [14-16]. Several studies have found associations between diabetes and greater odds of adverse clinical outcomes in COVID-19 including longer length of hospitalization, higher mortality rate, ICU admission, and mechanical ventilation $[4,5,9,13,17-19]$. Of note, patients with type 2 diabetes mellitus (T2D) with wellcontrolled blood glucose (BG) levels had lower mortality rates compared to those with poor BG control [17]. Regarding glucose-lowering medications, little is known about their impact on the characteristics and prognosis of patients with T2D and COVID-19. Recent data indicated that insulin users had a greater risk of poor prognosis and in-hospital mortality [5]. However, these observations may also be explained by confounders, as insulin use is associated with greater severity, duration of disease, and the presence of more complications. The relationship between T2D and COVID-19 outcomes warrants further characterization $[20,21]$. Further assessment of patient clinical characteristics and medication usage may inform treatment strategies and risk stratification efforts for patients with T2D and COVID-19.

Although studies have reported that patients with diabetes are a high-risk group for developing severe disease, the clinical and demographic characteristics as well as specific risk factors of patients with diabetes with severe forms of COVID-19 in the US have not been 
extensively studied. Further studies are needed to identify current practice patterns that may mitigate severe disease. Here, we evaluate risk factors associated with COVID-19 hospitalization and in-hospital mortality in a nationwide, commercially insured, and Medicare Advantage population, overall and among people with T2D in the US. To our knowledge, this is the first study integrating multiple nationwide US databases to evaluate the risk of COVID-19 hospitalization and mortality among people with T2D.

\section{METHODS}

\section{Data Sources}

This was a retrospective cohort study examining individuals who either tested positive for SARSCoV-2 or were hospitalized for COVID-19. This study utilized administrative claims data from the UnitedHealth Group (UHG) Clinical Discovery Database. The UHG Clinical Discovery Database contains historic Medicare Advantage and commercial eligibility and insurance claims data as well as more real-time data streams providing SARS-CoV-2 laboratory test result data and clinician-confirmed COVID-19 inpatient admissions.

The SARS-CoV-2 laboratory test results were provided by select health systems, clinics, and third-party laboratories. Notably, as the laboratory data are provided from select sources, the SARS-CoV-2 tests are not comprehensive for the commercial and Medicare Advantage enrollees contained in the claims database and, as such, should not be used to determine the proportion of people infected with SARS-CoV-2 that were ultimately hospitalized. The COVID-19 inpatient admission data are triggered on hospital admission for suspected COVID-19 and include demographics, admission and discharge dates, and disposition of individuals with confirmed COVID-19 following a clinician's review (Supplementary Material). It therefore provides realtime disposition data and a complete view of COVID-19-related hospitalizations for the considered subset of Medicare Advantage and commercial health plan enrollees. See
Supplementary Material for additional details on this data source and data quality.

\section{Time Period}

While the COVID-19 inpatient admission data and the SARS-CoV-2 laboratory test data contain recent data, medical and pharmacy claims in UHG Clinical Discovery Database lag with the claims billing cycle. Due to this lag, baseline covariates utilizing claims data were identified in 2019 to make sure the claims datasets were complete. Medical comorbidities were identified through historical claims from January 1, 2019, through December 31, 2019, while prescription data were sourced from the pharmacy claims data from the last 6 months of 2019 as they were the most recently available medication data at the time of the analysis. Laboratory test data were used to identify individuals who tested positive for SARS-CoV-2, which included tests starting from March 9, 2020, through July 2 , 2020. The COVID-19 inpatient admission data included admissions between January 15, 2020, and July 15, 2020.

To ensure any potential hospitalization of an individual was captured and observed in the data, the observation period for inclusion in the eligible SARS-CoV-2 ${ }^{+}$test cohort was cut off 14 days prior (July 2) to the end of the observation period for inclusion in the COVID-19 inpatient cohort (July 15). This time window was selected to maximize the likelihood that a COVID-19 hospitalization following a positive SARS-CoV-2 laboratory test was detected and not prematurely censored because of lack of sufficient follow-up.

\section{Study Population}

Individuals included in the study population were $\geq 18$ years of age as of January 1, 2019, with 12 months of continuous enrollment in both medical and pharmacy coverage for calendar year 2019 to allow for a more complete medical claims history, which provides information regarding patients' recorded medical comorbidities and prescription claim history. In an attempt to create a cohort that had been 
exposed to and infected with COVID-19, inclusion required that individuals were either hospitalized because of COVID-19 or had a positive laboratory result for SARS-CoV-2 (including PCR, antigen, or antibody tests) during 2020. Any individual with a COVID-19 hospitalization, regardless of presence in the SARSCoV-2 laboratory test cohort, was considered in the COVID-19 hospitalized cohort, not both. Pregnant women and those with a diagnosis of gestational diabetes were excluded.

Hospitalization and in-hospital mortality outcomes were analyzed overall and in a subset of individuals diagnosed with T2D (Fig. 1). Detailed selection criteria can be found in the Supplementary Material.

\section{Outcomes}

For the purposes of the analyses in this study, the COVID-19 inpatient admission data were utilized to identify individuals hospitalized with

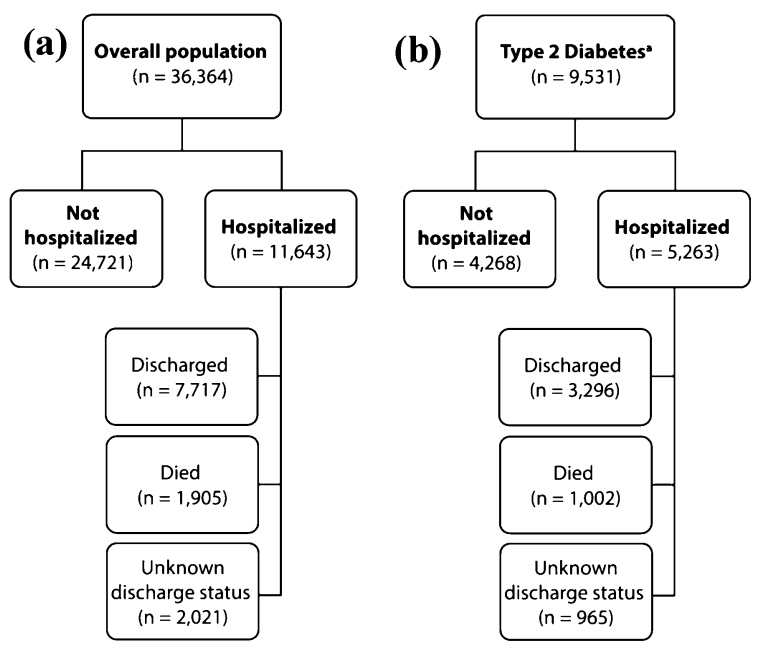

Fig. 1 Study population (SARS-CoV-2 $2^{+}$or COVID-19 hospitalized individuals). COVID-19 hospitalized individuals are further broken into subgroups based on their discharge status as discharged, died or unknown discharge status. Our dataset represents a subset of all SARS-CoV-2 testing, and therefore cannot be used to determine the proportion of people infected with SARS-CoV-2 that were hospitalized. a Overall population b Patients with Type 2 Diabetes. ${ }^{2}$ People with T2D are a subset of the overall population COVID-19 Coronavirus Disease-2019; $n$ number of individuals; SARS-CoV-2 severe acute respiratory syndrome coronavirus-2; $T 2 D$ type 2 diabetes mellitus
COVID-19. In-hospital mortality was based on the disposition provided in the inpatient dataset. For the in-hospital mortality outcome analysis, only known disposition (i.e., "discharged" or "died") was included and individuals still hospitalized at the time of study end were excluded.

\section{Baseline Demographics and Comorbidities}

Baseline demographic characteristics evaluated were age, sex, race/ethnicity, median household income category based on zip code tabulation area, and geographic region. Comorbidity indices including the Charlson Comorbidity Index (CCI) $[22,23]$ and Diabetes Complications Severity Index (DCSI) [24-27] were calculated. Baseline comorbidities were evaluated using ICD-10-CM (International Classification of Diseases, Tenth Revision, Clinical Modification) diagnosis codes in claims measured during calendar year 2019 for each index component of the CCI and DCSI (Supplementary Material). All CCI and DCSI comorbidities were considered originally as potential covariates in main regression models as well as in subgroup and sensitivity analysis models. In instances where CCI and DCSI comorbidities had significant overlap, we removed the CCI comorbidities (e.g., cerebrovascular disease) from the models. Additionally, the CCI comorbidity "diabetes with complications" was removed due to overlap with all DCSI comorbidities.

For people with T2D, diabetes medications were assessed at the class level and identified by a prescription fill during the last 6 months of 2019 for the following: biguanides, sulfonylureas, thiazolidinediones, glucagon-like peptide-1 receptor agonists, sodium-glucose cotransporter-2 (SGLT-2) inhibitors, dipeptidyl peptidase- 4 inhibitors, and insulins. The biguanides class is comprised entirely of metformin. When combination medications were used, each component of the medication was assigned to its corresponding drug class separately. No minimum day supply was required for the initial analyses with sensitivity analyses subsequently performed varying this requirement. In the overall population models, 
diabetes-specific drugs were removed to minimize collinearity with T2D.

\section{Statistical Analyses}

Descriptive analyses were used to evaluate characteristics of each study group, compare variables of interest (their counts and percent distributions between the groups), and determine which variables had large enough sample sizes to be considered for further analysis. Logistic regression with backward selection was used to model the outcomes of hospitalization and in-hospital mortality in the context of associated factors for each study population. The process of backward selection [28] began with the full list of covariates as considered in the full model, then removed one covariate at each step to maximize the reduction in Akaike's Information Criterion (AIC), and stopped where the AIC could no longer be reduced.

To address potential bias in the data and to understand robustness of observed associations of potential factors with hospitalization outcome, subgroup and sensitivity analyses were performed for the T2D population. Subgroup analyses were performed to control for disease severity by looking at different subsets of the study population such as (1) Medicare, (2) individuals with a baseline glycated hemoglobin in data (HbA1c; and controlling for HbA1c), and (3) individuals with at least one diabetes medication class other than biguanides. Sensitivity analyses were performed to control for drug exposure by requiring a minimum of 90and 180-day supply of medication during the baseline period (Supplementary Material).

Statistical computing was done with $\mathrm{R}$ version 3.6.2. Logistic regression models were fit using the "glm" function of the base package "stats" (Version 3.6.2). All results were considered statistically significant based upon an a priori designation of $p<0.05$.

\section{Compliance with Ethics Guidelines}

The study was approved by OptumLabs at UnitedHealth Group and Eli Lilly and Company. Because no identifiable protected health information was extracted or accessed during the course of the study, and all data were accessed in compliance with the Health Insurance Portability and Accountability Act's rules, Institutional Review Board approval or waiver of authorization was not required. OptumLabs at UnitedHealth Group granted permission for the Clinical Discovery Database to be used for the study.

\section{RESULTS}

In the overall population, we identified 11,643 individuals who were hospitalized because of COVID-19, and 24,721 individuals who were SARS-CoV $-2^{+}$but were not hospitalized. Among those with T2D, there were 5263 people who were hospitalized for COVID-19 and 4,268 people who were SARS-CoV-2 ${ }^{+}$but were not hospitalized (Fig. 1).

\section{Baseline Demographics and Comorbidities}

The mean age \pm standard deviation (SD) of the overall population was $70.1 \pm 15.1$ years (hospitalized) and $49.7 \pm 18.8$ years (not hospitalized). The mean age \pm SD of people with T2D was $71.6 \pm 12.5$ years (hospitalized) and $65.1 \pm 13.6$ years (not hospitalized). Both the overall and T2D population had an almost even distribution of males and females (Table 1).

In the T2D population, the majority of individuals were Caucasian (among non-missing; $54 \%$ of hospitalized; $55 \%$ of not hospitalized), lived in the Northeast (30\% of hospitalized; $42 \%$ of not hospitalized) or South (47\% of hospitalized; $38 \%$ of not hospitalized), and were enrolled in Medicare Advantage plans (87\% of hospitalized; $65 \%$ of not hospitalized; Table 1). Of the $36 \%$ and $48 \%$ of hospitalized and not hospitalized individuals with available HbA1c data, the average HbA1c levels at baseline for people with T2D were $7.2 \%(n=1876)$ and $7.0 \%(n=2069)$, respectively (Table 1$)$.

Overall, the prevalence of most comorbidities was higher among people with T2D compared to the overall population (Table 1, Supplemary Table S1). Additionally, people with T2D who were hospitalized had 
Table 1 Baseline demographic characteristics, comorbidities, and medications

\begin{tabular}{|c|c|c|c|c|}
\hline & \multicolumn{2}{|l|}{ Overall population } & \multicolumn{2}{|c|}{ Diabetes $(T 2 D)^{a}$} \\
\hline & $\begin{array}{l}\text { Not hospitalized } \\
(N=\mathbf{2 4 , 7 2 1})\end{array}$ & $\begin{array}{l}\text { Hospitalized } \\
(N=11,643)\end{array}$ & $\begin{array}{l}\text { Not } \\
\text { hospitalized } \\
(N=4268)\end{array}$ & $\begin{array}{l}\text { Hospitalized } \\
(N=5263)\end{array}$ \\
\hline Age in years, mean (SD) & $49.7(18.8)$ & $70.1(15.1)$ & $65.1(13.6)$ & $71.6(12.5)$ \\
\hline$<65$ years, $n(\%)$ & $18,223(74)$ & $3451(30)$ & $1750(41)$ & $1293(25)$ \\
\hline$\geq 65$ years, $n(\%)$ & $6498(26)$ & $8192(70)$ & $2518(59)$ & $3970(75)$ \\
\hline \multicolumn{5}{|l|}{ Sex, $n(\%)$} \\
\hline Female & $12,404(50)$ & $6207(53)$ & $2195(51)$ & $2842(54)$ \\
\hline Male & $12,317(50)$ & $5436(47)$ & $2073(49)$ & $2421(46)$ \\
\hline \multicolumn{5}{|l|}{ Race } \\
\hline Caucasian & $4421(18)$ & $5580(48)$ & $1533(36)$ & $2464(47)$ \\
\hline African American & $1292(5)$ & $2586(22)$ & $704(16)$ & $1616(31)$ \\
\hline Asian & $215(1)$ & $164(1)$ & $115(3)$ & $91(2)$ \\
\hline Hispanic & $555(2)$ & $440(4)$ & $275(6)$ & $248(5)$ \\
\hline Other $^{\mathrm{b}}$ & $356(1)$ & $272(2)$ & $153(4)$ & $131(2)$ \\
\hline Missing $^{c}$ & $17,882(72)$ & $2601(22)$ & $1488(35)$ & $713(13)$ \\
\hline \multicolumn{5}{|l|}{ Geographic region, $n(\%)$} \\
\hline Midwest & $2367(10)$ & $2054(18)$ & $287(7)$ & $788(15)$ \\
\hline West & $3956(16)$ & $1135(10)$ & $559(13)$ & $411(8)$ \\
\hline Northeast & $8538(35)$ & $3407(29)$ & $1782(42)$ & $1584(30)$ \\
\hline South & $9860(40)$ & $5047(43)$ & $1640(38)$ & $2480(47)$ \\
\hline Median household income, average & $\$ 70,934$ & $\$ 59,905$ & $\$ 61,544$ & $\$ 56,640$ \\
\hline \multicolumn{5}{|l|}{ Insurance plan type, $n(\%)$} \\
\hline Commercial & $17,858(72)$ & $2587(22)$ & $1479(35)$ & $705(13)$ \\
\hline Medicare & $6863(28)$ & $9056(78)$ & $2789(65)$ & $4558(87)$ \\
\hline \multicolumn{5}{|l|}{ Charlson comorbidities ${ }^{\mathrm{d}}, n(\%)$} \\
\hline Charlson Comorbidity Index (CCI), mean (SD) & $0.96(1.82)$ & $3.05(2.76)$ & $3.28(2.41)$ & $4.59(2.69)$ \\
\hline Chronic pulmonary disease & $2447(10)$ & $2638(23)$ & $726(17)$ & $1327(25)$ \\
\hline Congestive heart failure & $1064(4)$ & $2470(21)$ & $554(13)$ & $1492(28)$ \\
\hline Diabetes mellitus with chronic complications ${ }^{\mathrm{e}}$ & $1705(7)$ & $2890(25)$ & $1705(40)$ & $2890(55)$ \\
\hline Diabetes mellitus without chronic complications ${ }^{\mathrm{c}}$ & $4089(17)$ & $5016(43)$ & $4089(96)$ & $5016(95)$ \\
\hline Renal disease & $1311(5)$ & $2677(23)$ & $768(18)$ & $1705(32)$ \\
\hline \multicolumn{5}{|l|}{ Diabetes-related comorbidities $^{\mathrm{d}}, n(\%)$} \\
\hline $\begin{array}{l}\text { Diabetes Complication Severity Index (DCSI), mean } \\
\text { (SD) }\end{array}$ & $0.55(1.20)$ & $1.91(1.93)$ & $1.70(1.86)$ & $2.64(2.09)$ \\
\hline Cardiovascular disease & $3700(15)$ & $5423(47)$ & $1520(36)$ & $2850(54)$ \\
\hline Nephropathy & $1538(6)$ & $2923(25)$ & $1003(24)$ & $1969(37)$ \\
\hline
\end{tabular}


Table 1 continued

\begin{tabular}{|c|c|c|c|c|}
\hline & \multicolumn{2}{|l|}{ Overall population } & \multicolumn{2}{|l|}{ Diabetes $(T 2 D)^{a}$} \\
\hline & Not hospitalized $(N=24,721)$ & $\begin{array}{l}\text { Hospitalized } \\
(N=11,643)\end{array}$ & $\begin{array}{l}\text { Not hospitalized } \\
(N=4268)\end{array}$ & Hospitalized $(N=5263)$ \\
\hline Neuropathy & $1587(6)$ & $2013(17)$ & $1023(24)$ & $1646(31)$ \\
\hline Peripheral vascular disease & $1566(6)$ & $2832(24)$ & $969(23)$ & $1901(36)$ \\
\hline \multicolumn{5}{|l|}{ Diabetes medications, $n(\%)$} \\
\hline Biguanides & $2183(9)$ & 2067 (18) & $2183(51)$ & $2067(39)$ \\
\hline Sulfonylureas & $794(3)$ & $1063(9)$ & $794(19)$ & $1063(20)$ \\
\hline Thiazolidinediones & $179(1)$ & $204(2)$ & $179(4)$ & $204(4)$ \\
\hline GLP-1 receptor agonists & $358(1)$ & $417(4)$ & $358(8)$ & $417(8)$ \\
\hline DPP-4 inhibitors & $458(2)$ & $636(5)$ & $458(11)$ & $636(12)$ \\
\hline SGLT-2 inhibitors & $324(1)$ & $262(2)$ & $324(8)$ & $262(5)$ \\
\hline Long- and intermediate-acting insulins & $671(3)$ & $1361(12)$ & $671(16)$ & $1361(26)$ \\
\hline Short- and rapid-acting insulins & $423(2)$ & $1006(9)$ & $423(10)$ & $1006(19)$ \\
\hline No diabetes medication fills & $21,854(88)$ & $8064(69)$ & $1401(33)$ & $1684(32)$ \\
\hline \multicolumn{5}{|l|}{ Laboratory values } \\
\hline HbAlc value flag, $n(\%)$ & $5643(23)$ & $2720(23)$ & $2069(48)$ & $1876(36)$ \\
\hline HbAlc average & 6.0 & 6.7 & 7.0 & 7.2 \\
\hline
\end{tabular}

Our dataset represents a subset of all SARS-CoV-2 testing and therefore cannot be used to determine the proportion of people infected with SARS-CoV-2 that were hospitalized

COVID-19 coronavirus disease-2019; DPP-4 dipeptidyl peptidase-4; GLP-1 glucagon-like peptide-1; HbAIc glycated hemoglobin; ICD-10 International Classification of Diseases, Tenth Revision; $N$ number of individuals; $S A R S$ - $C o V-2$ severe acute respiratory syndrome coronavirus-2; $S D$ standard deviation; $S G L T-2$ sodium-glucose cotransporter-2; T2D type 2 diabetes mellitus

${ }^{a}$ People with T2D are a subset of the overall population

b Others include Native American, other, and unknown race

c "Missing" is for all commercial individuals

d Comorbidities present in $\geq 25 \%$ in at least one group

c The Charlson Comorbidity Index components Diabetes Mellitus Without Chronic Complications and Diabetes Mellitus with Chronic Complications are coded by mutually exclusive sets of ICD-10 codes. However, the observed proportion of people with diabetes in each of these groups does not sum to $100 \%$ as some individuals had care encounters corresponding to both sets of ICD-10 codes during the observation period

numerically higher mean CCI (4.59 vs. 3.28) and DCSI (2.64 vs. 1.70) scores compared with those with T2D who were not hospitalized (Table 1). Increased prevalence of cardiovascular disease (54\% vs. $36 \%$ ), nephropathy (37\% vs. $24 \%)$, neuropathy (31\% vs. $24 \%)$, and peripheral vascular disease ( $36 \%$ vs. $23 \%$ ) was observed for the people with T2D who were hospitalized compared to those who were not (Supplementary Table S1). Similar trends were observed for the overall population in terms of baseline demographics and comorbidities (Table 1).
Among diabetes medications in the T2D population, the largest proportion of individuals had a prescription fill for biguanides, which was also higher among those who were not hospitalized compared to those who were (51\% vs. $39 \%$, Table 1). The proportion of individuals who filled long- and intermediate-acting insulins $(26 \%$ vs. $16 \%)$ and short- and rapid-acting insulins (19\% vs. 10\%) were higher among hospitalized patients compared to those who were not (Table 1). 


\section{Factors Associated with Hospitalization}

For the overall population, age [odds ratio (OR): 1.05 95\% confidence interval (CI): 1.05-1.05)] and male sex (OR: 1.29; 95\% CI 1.22-1.36) were associated with significantly greater odds of hospitalization, where each 1-year increase in age was associated with a $5 \%$ increase in the odds of COVID-19 hospitalization. Comorbidities such as T2D, chronic pulmonary disease, congestive heart failure, dementia, paraplegia, metastatic tumor, metabolic disease, and neuropathy were associated with increased odds of hospitalization, with a particularly pronounced effect observed for dementia (OR: 2.42; 95\% CI 2.20-2.67) and paraplegia (OR: 2.54; 95\% CI 2.03-3.20) (Fig. 2a, Supplementary Table S3).

Among people with T2D, a similar trend was observed compared to the overall population except for liver disease, rheumatologic disease, and cardiovascular disease, which either did not remain in the model or were not significantly associated. Among diabetes medications, biguanides (OR: 0.78; 95\% CI 0.71-0.86) were associated with significantly lower odds of hospitalization, while sulfonylureas (OR: 1.29; 95\% CI 1.15-1.44), long-acting insulin (OR: 1.34 ; $95 \%$ CI $1.18-1.53$ ), and short- and rapidacting insulin (OR: 1.31; 95\% CI 1.12-1.53) were found to be associated with greater odds of hospitalization. Additional medications of interest that remained in the model were anticoagulants/antiplatelets and immunosuppressants, among which anticoagulants/ antiplatelets were associated with significantly greater odds of hospitalization (OR: 1.26; 95\% CI 1.11-1.42) (Fig. 2a, Supplementary Table S3).

\section{Factors Associated with Mortality}

We identified 9622 people in the overall population who were either discharged $(n=7717)$ from the hospital or died $(n=1905)$ in the hospital. Of those with T2D who were hospitalized, 4298 people were either discharged $(n=3296) \quad$ or died $(n=1002 ; \quad$ Fig. 1$)$. The resulting model output after the stepwise backward selection procedure is shown in Fig. 2b and Supplementary Table S4.
In the overall hospitalized population, age (OR: 1.04; 95\% CI 1.03-1.04), male sex (OR: 2.02; 95\% CI 1.81-2.25), and the Northeast region (OR: $1.75 ; 95 \%$ CI 1.51-2.04) relative to the Midwest were found to be significantly associated with increased odds of mortality. Significant comorbidities associated with mortality included T2D, leukemia/lymphoma, cardiovascular disease, nephropathy, and peripheral vascular disease (Fig. 2b, Supplementary Table S4).

In the case of the T2D population, a similar trend was observed for demographic variables. Significant comorbidities associated with mortality included chronic pulmonary disease (OR: 1.41; 95\% CI 1.19-1.66) and peripheral vascular disease (OR: 0.79; 95\% CI 0.67-0.93). Among diabetes medications, short- and rapid-acting insulins (OR: 1.21; 95\% CI 1.00-1.45) were found to be significantly associated with increased odds of mortality (Fig. 2b, Supplementary Table S4).

\section{Subgroup and Sensitivity Analyses}

Subgroup analyses performed using different subgroups of the T2D study population provided similar results to that of the full T2D population hospitalization model (Tables 2, S5). Sensitivity analyses focused on hospitalization because, as compared to the mortality models, model fit was better and more medications were significantly associated with hospitalization. In a subset of the T2D study groups for which $\mathrm{HbA1c}$ values were available, the HbA1c value was identified as a significant factor associated with hospitalization, where a one-unit increase in HbA1c was associated with a $10 \%$ increase in the odds of COVID-19 hospitalization (Table 2). Diabetes medications remained significant risk factors across all subgroups with ORs consistent with the findings in the model for the full T2D population, except the HbA1c subgroup where sulfonylureas and short- and rapid-acting insulins did not meet the threshold for significance. Among people with T2D who filled a prescription for a diabetes medication other than biguanides, prescription for SGLT-2 inhibitors emerged as an additional factor that was 


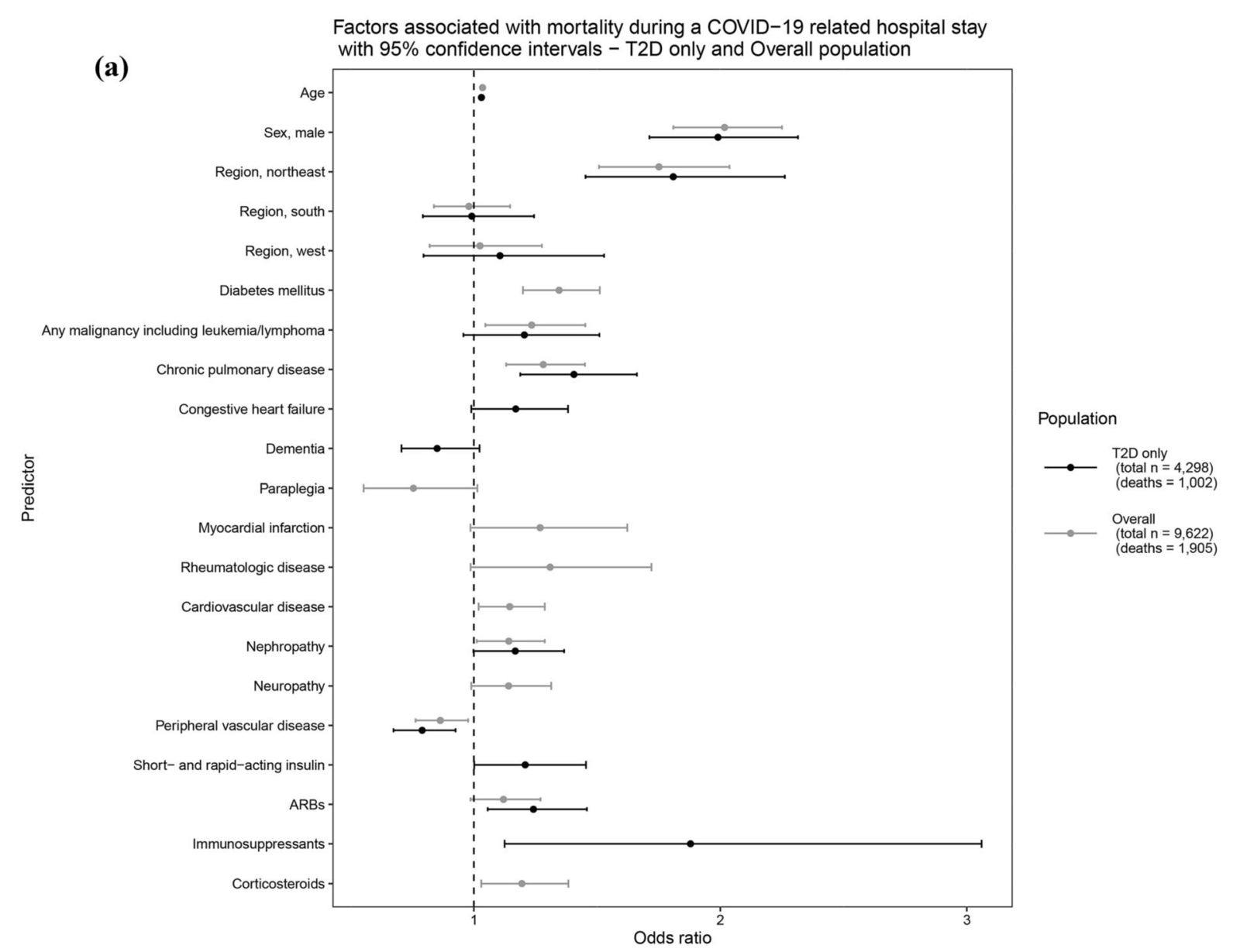

Fig. 2 Factors associated with (a) COVID-19 related hospitalization and (b) mortality subsequent to COVID19 related hospitalization overall population and T2D only. Example interpretation: Factors shown here with both black and gray lines were chosen by backwards selection for T2D only and overall population models, respectively. For a selected factor, overlap in black and gray lines and the position of mean odds ratio (dot in the middle of a line) as above $(\mathrm{OR}>1)$ or below $(\mathrm{OR}<1)$ the dashed line indicate that the magnitude and direction

associated with lower odds of hospitalization (Table 2).

Similarly, sensitivity analysis was performed in the T2D study population for the hospitalization outcome using two different revised T2D definitions and two different thresholds for days of medication supply. These analyses provided consistent results for the demographics, comorbidities, and diabetes medications compared to the hospitalization models for the full of the factor's association is consistent across T2D only and overall population models. Factors with only one line were either considered only in the corresponding model (e.g., diabetes medication classes were only considered in T2D only population models) or chosen by backwards selection in one of the models (e.g., antihypertensives remained only in the overall population model for hospitalization outcome). COVID-19 coronavirus disease2019; $n$ number of individuals; $O R$ odds ratio; T2D type 2 diabetes mellitus

T2D population and other subgroups. (Supplementary Table S6).

Figure 3 summarizes the consistency of diabetes medication associations with COVID-19related hospitalization across all models for T2D population; green shades indicate association with lower odds of hospitalization, and orange shades indicate association with greater odds of hospitalization. 


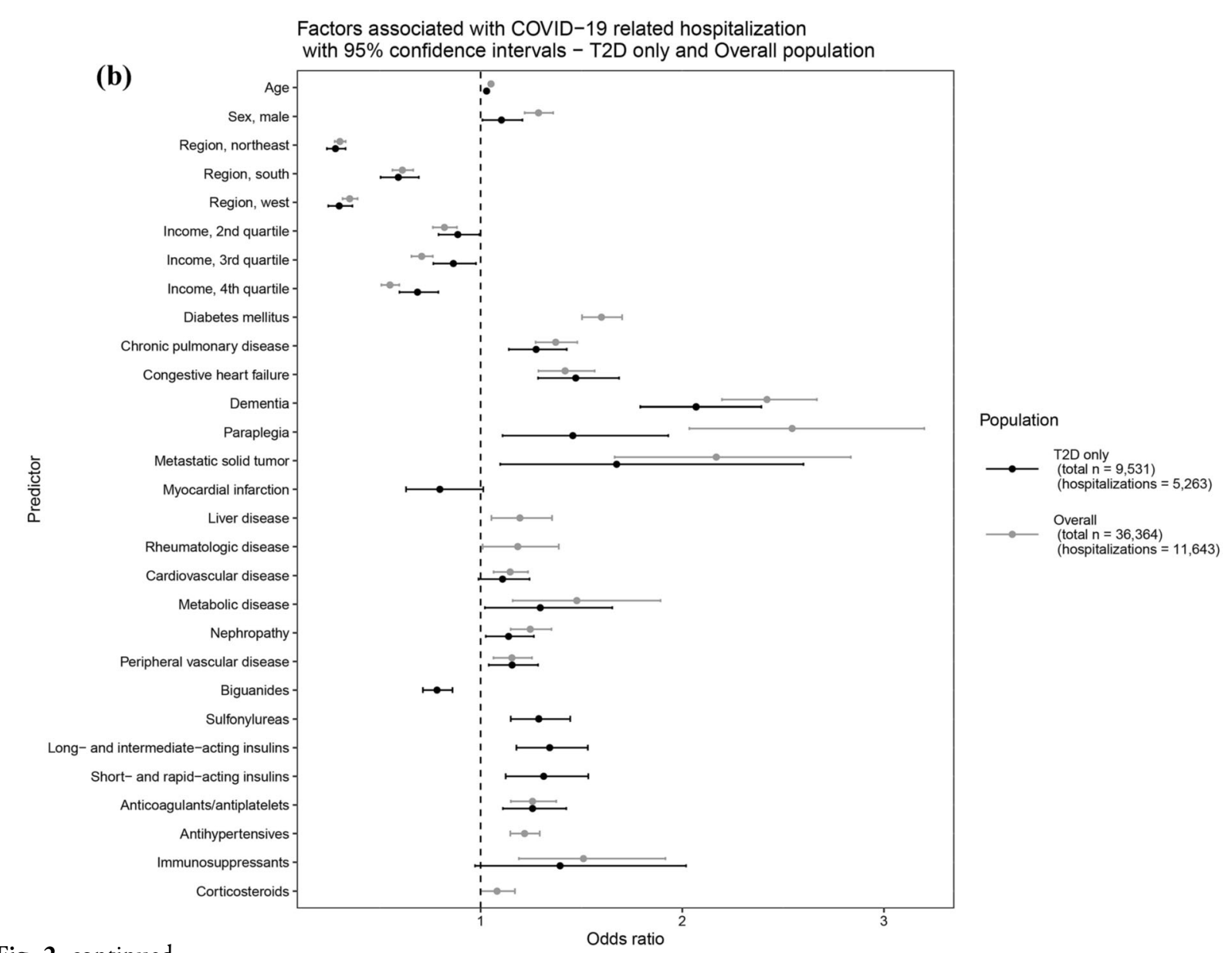

Fig. 2 continued

\section{DISCUSSION}

The outbreak of COVID-19 rapidly became a pandemic and caused a global health crisis. Significant health effects (including death and morbidity associated with hospitalization) were observed and seemed to preferentially affect older adults and those with comorbidities [6-13]. Research has demonstrated that comorbidities such as diabetes are important risk factors contributing to COVID-19 outcomes [14-16].

This study utilized a unique combination of datasets within the UHG Clinical Discovery Database, pairing historical medical and pharmacy claims with more real-time COVID-19 inpatient admission data and SARS-CoV-2 laboratory test data, to create a cohort of SARS$\mathrm{CoV}-2^{+}$individuals. We evaluated the associations of COVID-19 hospitalization and inpatient mortality with baseline demographics, comorbidities, and medications. Ultimately, the model fits were better for hospitalization than for in-hospital mortality, presumably because of unmeasured hospitalization-related factors (e.g., ventilation, treatment regimens). Risk factors for hospitalization and mortality in the overall population were generally consistent with those identified among individuals with T2D. In line with prior studies [5, 17], many of the findings suggest that indicators of more severe (or progressive) T2D were associated with worse outcomes, for example, higher HbA1c and use of diabetes medications typically used later in disease progression (e.g., short- and long-acting insulins).

Consistent with prior research [29-31] in the T2D population, older age, male sex, higher 
Table 2 Factors associated with COVID-19-related hospitalization in people with T2D: Subgroup analysis outcomes

\begin{tabular}{|c|c|c|c|c|}
\hline \multirow[t]{2}{*}{ Factors } & \multicolumn{2}{|c|}{$\begin{array}{l}\text { Patients with available } \\
\text { HbA1c values }\end{array}$} & \multicolumn{2}{|c|}{$\begin{array}{l}\text { Users of at least one non- } \\
\text { biguanide diabetes medication }\end{array}$} \\
\hline & $\overline{\text { OR }(95 \% ~ C I)}$ & $p$ value & $\overline{\text { OR }(95 \% ~ C I)}$ & $p$ value \\
\hline Age & $1.04(1.03-1.05)$ & $<0.001$ & $1.02(1.02-1.03)$ & $<0.001$ \\
\hline Sex, male & $1.16(1.00-1.33)$ & 0.047 & - & - \\
\hline \multicolumn{5}{|l|}{ Geographic region (base level: Midwest) } \\
\hline Northeast & $0.23(0.17-0.30)$ & $<0.001$ & $0.31(0.24-0.39)$ & $<0.001$ \\
\hline South & $0.64(0.48-0.85)$ & 0.002 & $0.66(0.52-0.82)$ & $<0.001$ \\
\hline West & $0.40(0.28-0.57)$ & $<0.001$ & $0.33(0.25-0.44)$ & $<0.001$ \\
\hline \multicolumn{5}{|l|}{ Median household income (base level: $<\$ 45,300$ ) } \\
\hline$\$ 45,300-\$ 60,600$ & $0.82(0.68-0.98)$ & 0.028 & $0.82(0.70-0.96)$ & 0.016 \\
\hline$\$ 60,600-\$ 83,100$ & $0.90(0.74-1.10)$ & 0.314 & $0.88(0.74-1.05)$ & 0.163 \\
\hline$\$ 83,100-\$ 250,000$ & $0.70(0.56-0.87)$ & 0.002 & $0.66(0.53-0.81)$ & $<0.001$ \\
\hline Any malignancy including leukemia/lymphoma & - & - & $1.24(0.99-1.55)$ & 0.060 \\
\hline Chronic pulmonary disease & $1.25(1.03-1.51)$ & 0.021 & $1.16(0.99-1.37)$ & 0.068 \\
\hline Congestive heart failure & $1.57(1.25-1.98)$ & $<0.001$ & $1.47(1.24-1.76)$ & $<0.001$ \\
\hline Dementia & $2.06(1.52-2.82)$ & $<0.001$ & $1.76(1.42-2.20)$ & $<0.001$ \\
\hline Paraplegia & $1.57(0.91-2.79)$ & 0.115 & $1.55(1.04-2.36)$ & 0.037 \\
\hline Myocardial infarction & - & - & $0.62(0.46-0.86)$ & 0.003 \\
\hline Metastatic solid tumor & $2.20(1.08-4.71)$ & 0.034 & - & - \\
\hline Cardiovascular disease & $1.19(1.00-1.41)$ & 0.055 & - & - \\
\hline Metabolic disease & $1.50(1.04-2.21)$ & 0.034 & - & - \\
\hline Nephropathy & $1.26(1.06-1.50)$ & 0.008 & $1.29(1.12-1.49)$ & $<0.001$ \\
\hline Neuropathy & $1.26(1.07-1.48)$ & 0.005 & - & - \\
\hline Biguanides & $0.84(0.72-0.97)$ & 0.019 & $0.74(0.65-0.85)$ & $<0.001$ \\
\hline Sulfonylureas & $1.18(0.99-1.40)$ & 0.068 & $1.19(1.03-1.37)$ & 0.019 \\
\hline SGLT-2 inhibitors & - & - & $0.82(0.68-0.99)$ & 0.038 \\
\hline Long- and intermediate-acting insulins & $1.34(1.08-1.67)$ & 0.008 & $1.30(1.13-1.51)$ & $<0.001$ \\
\hline Short- and rapid-acting insulins & $1.21(0.93-1.57)$ & 0.152 & $1.31(1.11-1.54)$ & 0.001 \\
\hline Antiplatelets & - & - & $1.24(1.05-1.47)$ & 0.011 \\
\hline Immunosuppressants & - & - & $1.51(0.92-2.57)$ & 0.112 \\
\hline Other antihypertensive ${ }^{a}$ & $1.23(1.07-1.42)$ & 0.004 & - & - \\
\hline Corticosteroids & $1.21(0.99-1.48)$ & 0.061 & - & - \\
\hline HbAlc value & $1.10(1.05-1.16)$ & $<0.001$ & - & - \\
\hline
\end{tabular}


Table 2 continued

\begin{tabular}{|c|c|c|c|c|}
\hline \multirow[t]{2}{*}{ Factors } & \multicolumn{2}{|c|}{$\begin{array}{l}\text { Patients with available } \\
\text { HbA1c values }\end{array}$} & \multicolumn{2}{|c|}{$\begin{array}{l}\text { Users of at least one non- } \\
\text { biguanide diabetes medication }\end{array}$} \\
\hline & OR (95\% CI) & $p$ value & OR (95\% CI) & $p$ value \\
\hline Observations, $n$ & 3916 & & 4641 & \\
\hline Hospitalizations, $n$ & 1861 & & 2767 & \\
\hline$R^{2}$ Tjur $^{\mathrm{b}}$ & 0.194 & & 0.139 & \\
\hline AUC & 0.754 & & 0.720 & \\
\hline
\end{tabular}

Example interpretation: A one-unit increase for $\mathrm{HbAlc}$ was associated with a $10 \%$ increase in the odds of being hospitalized because of COVID-19. Variables that did not remain at the end of the backward selection process are marked using a hyphen (-)

$A C E$ angiotensin-converting enzyme; $A R B$ angiotensin receptor blocker; $A U C$ area under the curve; $C I$ confidence interval; COVID-19 Coronavirus Disease-2019; HbAlc glycated hemoglobin; OR odds ratio; SGLT-2 sodium-glucose cotransporter-

2; $T 2 D$ type 2 diabetes mellitus

a Other antihypertensives include calcium-channel blockers and thiazide-like diuretics (i.e., any other first-line antihypertensive medications beyond ACE inhibitors and ARBs)

${ }^{\mathrm{b}} R^{2}$ Tjur metric provides the absolute value of difference between the average predicted probability of outcome for true positive subjects and the average predicted probability of outcome for true-negative subjects. $R^{2}$ Tjur is bounded between 0 and 1 , and a value close to 1 implies a better model fit

HbA1c, established cardiovascular disease, and impaired renal function were significantly associated with increased hospitalization and mortality. Here, a 1-year increase in age and one-unit increase in $\mathrm{HbA1c}$ were associated with a $3 \%$ and $10 \%$ increased risk for COVID-19 hospitalization, respectively. Together, results of this study indicate that people with T2D who also have comorbidities such as dementia, metastatic tumor, congestive heart failure, paraplegia, and metabolic disease are at amplified risk of COVID-19-related hospitalization. Similar findings were observed in the overall population. These findings are largely consistent with Centers for Disease Control (CDC) recommendations where people with cancer, chronic pulmonary disease, heart failure, and T2D are considered at increased risk of severe illness from COVID-19 [32]. Also, dementia is considered a condition that may increase risk according to the CDC, which is consistent with the findings of this study [32]. The consistency of these findings should be taken into consideration while planning population health risk stratification strategies or targeted outreach campaigns, particularly as vaccine prioritization efforts are underway both nationally and globally.

We attempted to isolate the effect of diabetes medication classes to explore whether any existing therapies could potentially play a role in reducing COVID-19 severity. Among those with T2D, biguanides were consistently associated with lower odds of hospitalization, whereas sulfonylureas and insulins were associated with greater odds of hospitalization (Fig. 3). The observed associations for biguanides and insulin are in line with recent studies $[5,33,34]$. While the observed association between these medications and hospitalization may reflect diabetes disease severity, subgroup analyses attempting to control for markers of disease severity (e.g., restriction to a Medicare Advantage population, controlling for $\mathrm{HbA1c}$, age, comorbidities) did not eliminate the association. Additionally, sensitivity analyses using revised T2D definitions and varying durations of medication use also resulted in consistent findings. Only short- and rapid-acting insulins showed a significant association with increased in-hospital mortality (Supplementary Table S4), which may be explained by longer diabetes 


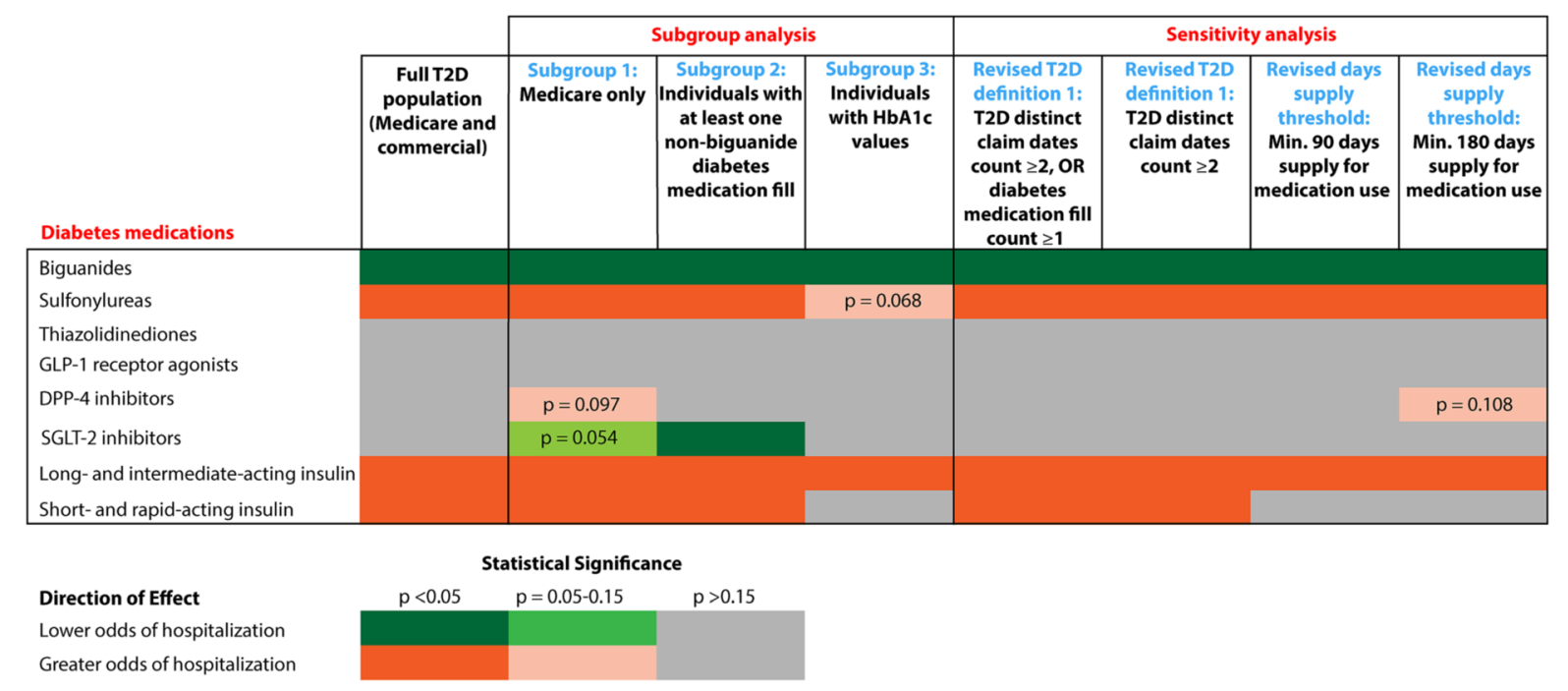

Fig. 3 Consistency of diabetes medication associations with COVID-19-related hospitalization across all models for T2D only population. Example interpretations: while still adjusting for baseline demographics, comorbidities, and other special interest medications for all subgroup and sensitivity analysis models as selected by the backward selection process, biguanides were consistently found to have statistically significant association with lower odds of hospitalization across all models. On the other hand, longand intermediate-acting insulin was consistently found to have statistically significant association with greater odds of hospitalization. Thiazolidinediones and GLP-1 receptor agonists were never found to have statistically significant associations with COVID-19-related hospitalization across all models for T2D population. SGLT-2 inhibitors were found to have statistically significant association with lower odds of hospitalization only for the Subgroup 2 model where the population is restricted to those individuals with at least one prescription fill of a non-biguanide diabetes medication. Subgroup 1: Included Race as an additional

duration and/or presence of complications. To date, there is limited evidence elucidating a mechanism through which biguanides confer benefit during SARS-CoV-2 infection. Preclinical work in in vitro and animal models in nonCOVID-19 and non-SARS-CoV-2 disease models suggests that biguanides could play a role in immunologic function through AMPK and mTOR pathways, though these mechanisms remain poorly understood in humans generally and in SARS-CoV-2 infection specifically $[35,36]$. Angiotensin receptor blockers (ARBs) predictor, which is available only for Medicare population. Subgroup 2: Intention was to focus on a higher severity diabetes group identified through medication categories. Medicare and commercial combined. Subgroup 3: Intention was to utilize $\mathrm{HbAlc}$ as an additional predictor to control for diabetes severity. Medicare and commercial combined. Sample size decreased by about 60\% (from $n=9531$ in full T2D population to $n=3916$ in Subgroup 3). Sensitivity analysis: Intention was to use a more conservative diabetes definition to see whether medication findings still hold. Current T2D definition of T2D diagnosis claim date count $>$ T1D diagnosis claim date count still holds. Time period: Medical claims and HbA1c data are from calendar year 2019; pharmacy claims data are from the last 6 months of 2019. COVID-19 coronavirus disease-2019; DPP-4 dipeptidyl peptidase-4; GLP-1 glucagon-like peptide-1; HbAlc glycated hemoglobin; $O R$ odds ratio; $S G L T-2$ sodium-glucose cotransporter2; $T 2 D$ type 2 diabetes mellitus

showed a significant association with increased in-hospital mortality (Supplementary Table S4). Observational studies of renin-angiotensin-aldosterone system inhibitors and association with COVID-19 outcomes have tended to be associated with neutral to reduced risk of COVID-19 hospitalization or mortality rather than increased risk as noted in our analyses $[37,38]$. However, most of the reviewed studies were not exclusively examining a T2D population, and limited prospective evidence on this topic remains. It has also been suggested that 
there may be an increased risk of COVID-19 mortality associated with patients receiving long-term treatment with ARBs that is due to the conditions associated with their use, including diabetes, rather than the drugs themselves [39]. Future research is needed that directly accounts for duration of T2D in the context of medication class effects.

\section{Limitations}

Findings in this retrospective cohort study are subject to several limitations. The study population contains only individuals with Medicare Advantage and commercial insurance plans. The SARS-CoV-2 laboratory tests are reported from select facilities and third-party vendors. Because it does not capture all outpatient tests, conclusions regarding the prevalence of hospitalization among different groups cannot be made.

Historical claims data are only available as the billing cycle permits, often lagging by 3-6 months, which restricted the time periods to identify medical diagnoses and pharmacy utilization. Also, in this study, no observations related to medication data were included before hospitalization (January 1, 2020-March 1, 2020) to ensure completeness of prior year medical and pharmacy claims data and to minimize the impact of pandemic-related reductions on 2020 care utilization in underestimating baseline medication exposure and comorbidity history. All events and comorbidities are limited to individuals seeking care and subsequently billing through insurance. Current data sources do not provide insight into in-hospital medications or treatment regimens, including ventilator status or level of care (e.g., intensive care unit), which are especially critical for mortality modeling. We also note the limitation that the data included in the study only covered up to July 2020, which would not account for any COVID-19 variants that have now emerged.

Pharmacy claims are limited to medications filled through an individual's insurance; therefore, medication information may be missing for individuals who are paying fully out of pocket for their medications. Notably, 32\% of people with T2D who were hospitalized in this study did not have any observed diabetes medication fill (Table 1), suggesting alternative methods of obtaining prescriptions or reliance on non-pharmacologic treatments like lifestyle modifications. Also, presence of a pharmacy claim is not equivalent to adherence.

Some important confounders of interest were not either available or well coded in medical claims data, such as diabetes duration and severity, obesity (and BMI), smoking, certain laboratory values of interest (e.g., eGFR), individual level income, occupation and SARS-CoV-2 exposure risk, living situation, and other social determinants of health. Regarding medication class associations, the inability to adjust for some of these confounders (e.g., diabetes duration) was addressed through subgroup analyses, which were used to attempt to control for markers of disease severity, but did not eliminate the association. Sensitivity analyses using revised T2D definitions and varying durations of medication use also resulted in consistent findings (Fig. 3). Additionally, race information was not available for the commercial population. Due to the high percentage of missingness, race was only considered in a subgroup analysis of the Medicare population (Supplementary Material). Notably, we did not control for several important pandemic confounders, such as time course and varying mortality and hospitalization rates, geographic trends, stay at home orders, and testing availability. We did include adjustments for renal function (severe renal disease from the CCI, nephropathy from the DCSI) based on claims-based measures (Table 1). Similarly, we attempted to characterize obesity (Table 1) with available claims-based evidence.

\section{CONCLUSIONS}

This is the first published study using a national database in the US to evaluate the risk of COVID-19 hospitalization and mortality among people with T2D. The study highlights the link between T2D and increased morbidity and mortality related to COVID-19, where patients with multiple chronic illnesses (e.g., dementia, metastatic tumor, congestive heart failure, paraplegia, and metabolic disease), male sex, 
and advanced age appear to be at highest risk. While recognizing the potential for disease progression confounding, biguanides usage was consistently associated with lower odds of hospitalization, whereas sulfonylurea and insulin usage were associated with higher odds of hospitalization. The findings were robust, remaining largely consistent across multiple sensitivity analyses. Findings of this study further support known at-risk populations and can help guide risk stratification efforts across population health strategies (e.g., vaccine prioritization or targeted outreach campaigns).

\section{ACKNOWLEDGEMENTS}

Funding. This work was supported by UnitedHealth Group and Eli Lilly and Company. Eli Lilly and Company funded the journal's Rapid Service Fee.

Medical Writing, Editorial, and Other Assistance. We thank Carlos Vallarino for his statistical consultation and Erin Walls for her overall contributions to the study initiation and coordination activities. The authors thank An Ting Jhuang, PhD, and Elaine Preimesberger for reviewing the manuscript and providing feedback on data summaries and statistical methodologies. The authors thank Ankit Turakhiya, PhD and Rhea Lewis, PhD, for providing writing and editorial support. Carlos Vallarino, Erin Walls, Ankit Turakhiya, and Rhea Lewis are employees and shareholders of Eli Lilly and Company. An-Ting Jhuang and Elaine Preimesberger are employees and shareholders of UnitedHealth Group.

Authorship. All named authors meet the International Committee of Medical Journal Editors (ICMJE) criteria for authorship for this article, take responsibility for the integrity of the work as a whole, and have given their approval for this version to be published.

Authorship Contributions. Lida Etemad, Callahan Clark, Elif Tokar Erdemir, Nathan Zimmerman, Abraham Reddy, Cody J. Lensing,
Kristina S. Boye, Emily R. Hankosky, Brian D. Benneyworth, Matan C. Dabora, M. Angelyn Bethel, Robert J Heine have contributed to the study concept and design. Ramira San Juan, Nathan Zimmerman, Abraham Reddy have contributed to the study conduct and data collection. Nathan Zimmerman, Abraham Reddy, Elif Tokar Erdemir, Callahan Clark, Cody J. Lensing, Scott Sailer, Lida Etemad have contributed to the data analysis. Callahan Clark, Elif Tokar Erdemir, Kristina S. Boye, Emily R. Hankosky, Brian D. Benneyworth have contributed to the manuscript writing. All authors contributed to critical revisions of the manuscript and have approved the final version of the manuscript submitted.

Disclosures. Kristina S. Boye, Brian D. Benneyworth, Matan C. Dabora, Emily R. Hankosky, M. Angelyn Bethel, and Robert J Heine are employees and shareholders of Eli Lilly and Company. Elif Tokar Erdemir, Nathan Zimmerman, Abraham Reddy, Callahan Clark, Cody J. Lensing, Scott Sailer, Ramira San Juan, and Lida Etemad are employees and shareholders of UnitedHealth Group.

Compliance with Ethics Guidelines. The study was approved by OptumLabs at UnitedHealth Group and Eli Lilly and Company. Because no identifiable protected health information was extracted or accessed during the course of the study, and all data were accessed in compliance with the Health Insurance Portability and Accountability Act's rules, Institutional Review Board approval or waiver of authorization was not required. OptumLabs at UnitedHealth Group granted permission for the Clinical Discovery Database to be used for the study.

Data Availability. The datasets generated during and/or analyzed during the current study are not publicly available but, under certain conditions, may be made available to editors and their approved auditors under a data use agreement to confirm the findings of the current study.

Open Access. This article is licensed under a Creative Commons Attribution- 
NonCommercial 4.0 International License, which permits any non-commercial use, sharing, adaptation, distribution and reproduction in any medium or format, as long as you give appropriate credit to the original author(s) and the source, provide a link to the Creative Commons licence, and indicate if changes were made. The images or other third party material in this article are included in the article's Creative Commons licence, unless indicated otherwise in a credit line to the material. If material is not included in the article's Creative Commons licence and your intended use is not permitted by statutory regulation or exceeds the permitted use, you will need to obtain permission directly from the copyright holder. To view a copy of this licence, visit http:// creativecommons.org/licenses/by-nc/4.0/.

\section{REFERENCES}

1. Li H, Liu SM, Yu XH, Tang SL, Tang CK. Coronavirus disease 2019 (COVID-19): current status and future perspectives. Int $\mathrm{J}$ Antimicrob Agents. 2020;55(5):105951.

2. WHO Director-General's opening remarks at the media briefing on COVID19. 2020. https://www. who.int/director-general/speeches/detail/whodirector-general-s-opening-remarks-at-the-mediabriefing-on-covid-19-11-march-2020. Accessed 20 Apr 2020.

3. World Health Organization. Coronavirus disease (COVID-2019) situation reports-90. 2021. https:// www.who.int/publications/m/item/weeklyepidemiological-update-2-february-2021. Accessed 05 Feb 2021.

4. Huang C, Wang Y, Li X, et al. Clinical features of patients infected with 2019 novel coronavirus in Wuhan, China. Lancet. 2020;395(10223):497-506.

5. Chen Y, Yang D, Cheng B, et al. Clinical characteristics and outcomes of patients with diabetes and COVID-19 in association with glucose-lowering medication. Diabetes Care. 2020;43(7):1399-407.

6. Centers for Disease Control and Prevention. Evidence used to update the list of underlying medical conditions that increase a person's risk of severe illness from COVID-19. 2020. https://stacks.cdc. gov/view/cdc/89840. Accessed 30 Aug 2020.
7. CDC COVID-19 Response Team. Preliminary estimates of the prevalence of selected underlying health conditions among patients with coronavirus disease 2019-United States, February 12-March 28, 2020. MMWR Morb Mortal Wkly Rep. 2020;69(13): $382-6$.

8. Chen N, Zhou M, Dong X, et al. Epidemiological and clinical characteristics of 99 cases of 2019 novel coronavirus pneumonia in Wuhan, China: a descriptive study. Lancet. 2020;395(10223):507-13.

9. Wu C, Chen X, Cai Y, et al. Risk factors associated with acute respiratory distress syndrome and death in patients with coronavirus disease 2019 pneumonia in Wuhan. China JAMA Intern Med. 2020;180(7):934-43.

10. Onder G, Rezza G, Brusaferro S. Case-fatality rate and characteristics of patients dying in relation to COVID-19 in Italy. JAMA. 2020;323(18):1775-6.

11. Petrilli CM, Jones SA, Yang J, et al. Factors associated with hospital admission and critical illness among 5279 people with coronavirus disease 2019 in New York City: prospective cohort study. BMJ. 2020;369:m1966.

12. Liu K, Fang YY, Deng Y, et al. Clinical characteristics of novel coronavirus cases in tertiary hospitals in Hubei Province. Chin Med J (Engl). 2020;133(9): 1025-31.

13. Grasselli G, Zangrillo A, Zanella A, et al. Baseline characteristics and outcomes of 1591 patients infected with SARS-CoV-2 admitted to ICUs of the Lombardy region, Italy. JAMA. 2020;323(16): 1574-81.

14. Richardson S, Hirsch JS, Narasimhan M, et al. Presenting characteristics, comorbidities, and outcomes among 5700 patients hospitalized with COVID-19 in the New York City area. JAMA. 2020;323(20):2052-9.

15. Zhou F, Yu T, Du R, et al. Clinical course and risk factors for mortality of adult inpatients with COVID-19 in Wuhan, China: a retrospective cohort study. Lancet. 2020;395(10229):1054-62.

16. Zhang JY, Shang T, Ahn D, et al. How to best protect people with diabetes from the impact of SARS-CoV2: report of the international COVID-19 and diabetes summit. J Diabetes Sci Technol. 2021. https:// doi.org/10.1177/1932296820978399.

17. Zhu L, She ZG, Cheng X, et al. Association of blood glucose control and outcomes in patients with COVID-19 and pre-existing type 2 diabetes. Cell Metab. 2020;31(6):1068-1077.e3. 
18. Bode B, Garrett V, Messler J, et al. Glycemic characteristics and clinical outcomes of COVID-19 patients hospitalized in the United States. J Diabetes Sci Technol. 2020;14(4):813-21.

19. Seiglie J, Platt J, Cromer SJ, et al. Diabetes as a risk factor for poor early outcomes in patients hospitalized with COVID-19. Diabetes Care. 2020;43(12): 2938-44.

20. Drucker DJ. Coronavirus infections and type 2 diabetes-shared pathways with therapeutic implications. Endocr Rev. 2020;41(3):bnaa011.

21. Bornstein SR, Rubino F, Khunti K, et al. Practical recommendations for the management of diabetes in patients with COVID-19. Lancet Diabetes Endocrinol. 2020;8(6):546-50.

22. Deyo RA, Cherkin DC, Ciol MA. Adapting a clinical comorbidity index for use with ICD-9-CM administrative databases. J Clin Epidemiol. 1992;45(6): 613-9.

23. Quan H, Sundararajan V, Halfon P, et al. Coding algorithms for defining comorbidities in ICD-9-CM and ICD-10 administrative data. Med Care. 2005;43(11):1130-9.

24. Klabunde CN, Potosky AL, Legler JM, Warren JL. Development of a comorbidity index using physician claims data. J Clin Epidemiol. 2000;53(12): 1258-67.

25. Chang HY, Weiner JP, Richards TM, Bleich SN, Segal JB. Validating the adapted Diabetes Complications Severity Index in claims data. Am J Manag Care. 2012;18(11):721-6.

26. Glasheen WP, Renda A, Dong Y. Diabetes Complications Severity Index (DCSI)-update and ICD-10 translation. J Diabetes Complicat. 2017;31(6): 1007-13.

27. Young BA, Lin E, Von Korff M, et al. Diabetes complications severity index and risk of mortality, hospitalization, and healthcare utilization. Am J Manag Care. 2008;14(1):15-23.

28. Hastie T, Tibshirani R, Friedman JH. The elements of statistical learning: data mining, inference, and prediction. 2nd ed. New York: Pringer; 2009.

29. Yang J, Zheng Y, Gou X, et al. Prevalence of comorbidities and its effects in patients infected with SARS-CoV-2: a systematic review and metaanalysis. Int J Infect Dis. 2020;94:91-5.

30. Jordan RE, Adab P, Cheng KK. Covid-19: risk factors for severe disease and death. BMJ. 2020;368:m1198.

31. Holman N, Knighton P, Kar P, et al. Risk factors for COVID-19-related mortality in people with type 1 and type 2 diabetes in England: a population-based cohort study. Lancet Diabetes Endocrinol. 2020;8(10):823-33.

32. Centers for Disease Control and Prevention (CDC). People with certain medical conditions. 2021. https://www.cdc.gov/coronavirus/2019-ncov/needextra-precautions/people-with-medical-conditions. html. Accessed 13 Jan 2021.

33. Singh AK, Singh R. Is metformin ahead in the race as a repurposed host-directed therapy for patients with diabetes and COVID-19? Diabetes Res Clin Pract. 2020;165:108268.

34. Yu B, Li C, Sun Y, Wang DW. Insulin treatment is associated with increased mortality in patients with COVID-19 and type 2 diabetes. Cell Metab. 2021;33(1):65-77.e2.

35. Ursini F, Russo E, Pellino G, et al. Metformin and autoimmunity: a "New Deal" of an old drug. Front Immunol. 2018;9:1236.

36. Sharma S, Ray A, Sadasivam B. Metformin in COVID-19: a possible role beyond diabetes. Diabetes Res Clin Pract. 2020;164:108183.

37. Xu J, Teng Y, Shang L, Gu X, Fan G, Chen Y, Tian R, Zhang S, Cao B. The effect of prior ACEI/ARB treatment on COVID-19 susceptibility and outcome: a systematic review and meta-analysis. Clin Infect Dis. 2021;72(11):e901-13.

38. Baral R, Tsampasian V, Debski M, Moran B, Garg P, Clark A, Vassiliou VS. Association between reninangiotensin-aldosterone system inhibitors and clinical outcomes in patients with COVID-19: a systematic review and meta-analysis. JAMA Netw Open. 2021;4(3):e213594.

39. Cugno M, Gualtierotti R, Casazza G, Tafuri F, Ghigliazza G, Torri A, et al. Mortality in PATIENTS with COVID-19 on renin angiotensin system inhibitor long-term treatment: an observational study showing that things are not always as they seem. Adv Ther. 2021;38(5):2709-16. 